\title{
Le sacrement de pénitence ou la Confession sous le Régime français (suite et fin)
}

\section{Valérien Roy}

Volume 16, numéro 4, mars 1963

URI : https://id.erudit.org/iderudit/302231ar

DOI : https://doi.org/10.7202/302231ar

Aller au sommaire du numéro

Éditeur(s)

Institut d'histoire de l'Amérique française

ISSN

0035-2357 (imprimé)

1492-1383 (numérique)

Découvrir la revue

Citer cet article

Roy, V. (1963). Le sacrement de pénitence ou la Confession sous le Régime français (suite et fin). Revue d'histoire de l'Amérique française, 16(4), 567-580. https://doi.org/10.7202/302231ar d'utilisation que vous pouvez consulter en ligne.

https://apropos.erudit.org/fr/usagers/politique-dutilisation/ 


\title{
LE SACREMENT DE PÉNITENCE OU LA CONFESSION SOUS LE RÉGIME FRANÇAIS *
}

\author{
(Suite et fin)
}

CHAPITRE II

\section{L'Excommunication.}

Cet ultime châtiment ecclésiastique fut assez souvent fulminé, sinon infligé de fait, durant le régime français, devant des abus concernant la traite de l'eau-de-vie et le mariage chrétien. Les enfants eux-mêmes apprenaient dans leur catéchisme qu'il y avait alors 9 commandements de l'Église, et que les deux derniers se formulaient ainsi:

8e: Les excommuniés fuïras, les dénoncés expressement.

9e: Quand excommuniés tu seras, fais-toi absoudre promptement. ${ }^{11}$

C'était laisser entendre que ces individus, exclus du sein de l'Église, pouvaient se rencontrer même parmi le peuple des villes et des campagnes, puisqu'on prescrivait de fuir leur compagnie. Le Rituel, les Catéchismes et les Mandements signalent les cas encourant l'excommunication, parce qu'ils doivent être reconnus et appris par tout le monde. Le Catéchisme St-Vallier énumère seulement trois cas de peine majeure ${ }^{12}$ :

1. Pour les garçons qui entrent dans les monastères de filles, nonobstant la défense de l'Eglise;

2. Pour les filles qui entrent dans les monastères de garçons ;

3. Pour ceux qui battent les prêtres, les religieux, et les insultent.

Le Catéchisme de Sens cite à peu près les mêmes cas ${ }^{13}$ : excommunication:

* Voir notre Revue, XVI : 225-239, 409-427.

11 Catéchisme St-Vallier, 232.

12 Loc. cit.

13 Catéchisme de Sens, 1751, 103. 
1. pour celui qui bat les ecclésiastiques et les religieux;

2. pour celui qui entre dans les Couvents de religieuses;

3. pour celui qui ne révèle pas, quand il le doit, ce qu'il sait touchant les Monitoires qui ont été publiés ;

4. pour celui qui ne communie pas à Pâques ;

5. pour celui qui désobéit aux Ordonnances des Evêques publiées sous peine d'excommunication.

Comme pratique, Mgr Languet invite à craindre l'excommunication, à s'interdire ce qui y fait tomber, à s'en faire promptement relever dans le besoin, à ne jamais parler du Pape et des Évêques qu'avec grand respect, à ne point en médire, ni souffrir qu'on en parle mal devant soi. ${ }^{14}$ Dans le Catéchisme Briand, les mêmes cas sont signalés. ${ }^{15}$

Depuis les temps de Mgr de Laval, l'usage de lancer des excommunications avait dû, par la force des choses, devenir plus courant. La fameuse question de l'eau-de-vie en fut l'une des causes les plus «diaboliques》. Le 5 mai 1660 , dès son second Mandement, Monseigneur fait, du troc de l'eau-de-vie et du vin, en échange des pelleteries des sauvages, un cas d'excommunication ${ }^{16}$. Dans sa Vie, on rappelle un cas entre plusieurs où il était monté en chaire, mître en tête et crosse en main, pour tonner contre cet abus et impliquer sous la même peine capitale tous les détracteurs à ses objurgations. ${ }^{17}$ Et de fait, il excommunie officiellement un nommé Pierre Aigron dit la Mothe, pour avoir traité des boissons enivrantes aux sauvages ${ }^{18}$. Devant les protestations des victimes de ses foudres, Monseigneur croit opportun de référer le cas aux savants Docteurs de la Sorbonne, afin d'avoir leur opinion sur les pouvoirs disciplinaires de l'Évêque en ce domaine. Le corps professoral répond à cette con-

14 Loc. cit.

15 Catéchisme Briand, 1782, 2ème éd., 131.

16 Mandements, I: $14 \mathrm{~s}$.

17 Gosselin, Vie de Mgr de Laval, II: 564, 6 mai 1660.

18 Excommunication contre Pierre... pour avoir traité des boissons enivrantes aux sauvages, 18 av. 1661, dans Mandements, I: 30ss. 
sultation, de la façon suivante: "L'Ordinaire ou Prélat peut défendre sous peine d'excommunication ipso facto aux Européens la vente de telles boissons. Délibéré à Paris, le ler février 1662. ${ }^{19}$ La loi de l'excommunication avait été suspendue en octobre 1661; mais devant les abus qui se multiplient, Mgr de Laval lève la suspense et remet en vigueur la même peine ultime. ${ }^{20}$ Et il revient encore sur la même défense un mois plus tard. ${ }^{21}$ En 1668, Mgr de Laval signale de nouveau le problème à l'attention du clergé et des missionnaires, mais cette fois, il laisse le tout au jugement du confesseur, leur rappelant que l'excommunication a été levée, mais que la chose reste grave; c'est à eux de voir ! ${ }^{22}$

Mgr Dosquet, devant les mêmes difficultés, publie un Mandement, en date du 26 novembre 1730, où il défend d'absoudre ceux qui directement ou indirectement contribuent à enivrer les sauvages, et en fait un péché à lui réservé. ${ }^{23}$ Un garde-magasin du fort Frontenac dut alors monter à Québec pour se faire absoudre; il n'était pas allé à confesse depuis 4 ans; à Québec, l'Évêque donne à un confesseur un billet, c'est-à-dire une permission par écrit de l'absoudre. ${ }^{24}$

Il était d'usage, sous le régime français, de faire du «charivari » à quelqu'un qui se remariait trop tôt après la mort de son conjoint, ou qui s'engageait dans le mariage à un âge vraiment tardif ! Cette coutume donnait lieu à bien des abus qui, par contrefaçon, tournaient souvent en ridicule les cérémonies du sacrement. Mgr de Laval prend occasion de l'un

19 Ibid., 41.

20 Excommunication contre ceux qui vendent de la boisson aux sauvages remise en force, le 24 février 1662, ibid., 42s.

21 Le 30 avril 1662 , ibid., $43 \mathrm{~s}$.

22 Le 9 fév. 1668, ibid., 72s.

${ }^{23}$ Gosselin, L'Eglise du Canada, V: 177; Mandements, I: 535. - Ce Mandement produisit une impression salutaire: les missionnaires en furent bien aises; les commandants de Forts furent obligés de se montrer plus sévères. Beauharnais et Hocquart commencèrent à se plaindre, mais Monseigneur les laissa épiloguer, se contentant d'en adoucir l'application par de sages tempéraments. A Paris, on lui parle de son Mandement, et le Ministre essaie en vain de le lui faire modifier.

${ }^{24}$ Gosselin, L'Eglise du Canada, V: 179. 
de ces charivaris pour lancer un Mandement dans lequel il déplore ces désordres à caractère païen. «Ayant été informé qu'en conséquence du mariage célébré dans cette ville de Québec depuis six jours, grand nombre de personnes de l'un et l'autre sexe se seraient assemblées toutes les nuits sous le nom de charivari et auraient dans leurs désordres et libertés scandaleuses, comme il arrive ordinairement, commis des actions impies et qui vont à une entière dérision de nos mystères, et des vérités de la Religion chrétienne et des plus saintes cérémonies de l'Église, ce qui nous aurait obligés de recourir au bras séculier pour faire cesser ces sortes d'assemblées, (...) nonobstant quoi nous avons appris (...) qu'ils vont en augmentant de jour en jour (...) ce qui nous oblige de joindre l'autorité de l'Église à celle du bras séculier. (...) Nous, pour ces causes, faisons très expresses inhibitions et défenses à tous les fidèles de notre diocèse de se trouver à aucune des dites assemblées qualifiées de charivari, aux pères et aux mères d'y envoyer ou permettre que leurs enfants y aillent, aux maîtres et maîtresses d'y envoyer leurs domestiques, le tout sous peine d'excommunication ... ${ }^{25}$

De même, le Mariage à la gaumine (ou gomine) était loin d'être selon les lois de l'Église. Il était contracté par deux parties, lesquelles, en présence du curé qui refuse de les marier, protestent qu'elles se prennent pour mari et femme, au cours de la célébration d'une messe nuptiale pour deux autres conjoints. Ceux qui contractaient de «si détestables mariages, » de même que les témoins apostés, les conseillers et les notaires, étaient passibles d'excommunication, réservée à l'Évêque. ${ }^{26}$ Dans la Vie de Mgr de St-Vallier, on relate longuement le cas fameux du

25 Mandement du 3 juillet 1683, I: 114s. Voir, Roy, op. cit., VII : 147s.

26 Cette mode était prohibée par les lois ecclésiastiques et civiles. Les Docteurs catholiques sont partagés sur sa validité intrinsèque. Ils le considèrent comme «quasi clandestin 》 et conseillent que les « mariés 》 fassent réhabiliter leur mariage devant l'Eglise. Un Curé ne pourrait donner un certificat à ceux qui se seraient unis sans avoir reçu la bénédiction nuptiale. En 1579, une ordonnance de Blois fait passer dans la législation un décret du Concile de Trente à ce sujet. Un certain Gaumon fut le premier homme à se marier de la sorte. Cet usage gagna la Nouvelle-France, dès le 18e siècle. La pratique était commune en 1717, comme le laisse entendre le Mandement de Mgr de St-Vallier, en date du 24 mai 1717. 
mariage à la gaumine de Paul de Montéléon; une affaire qui souleva toute une querelle. ${ }^{27}$

Un certain François Desjordy, capitaine réformé d'un détachement de la marine, résidant à Champlain, vivait en concubinage avec une femme de Batiscan. L'Évêque ordonne au curé de Batiscan de porter une sentence solennelle d'excommunication, au prône de la messe. ${ }^{28}$ Et ce d'Ailleboust des Musseaux, qui gardait chez lui une fille d'Agnier se voit refuser l'absolution, par ordre de Mgr de St-Vallier. ${ }^{29}$ Pour deux autres cas d'adultère, le même Evêque doit porter les mêmes peines..$^{30}$

Pour des infractions qui semblent de moindre importance, l'autorité religieuse inflige également cette peine d'exclusion du sein de l'Église. Par exemple, elle rappelle aux fidèles que, «selon le pouvoir que donne l'autorité du Concile de Trente (22e session), le 3e Concile de Constance, le 11e Canon du Concile de Sardaigne et le 21e Concile d'Elvire, l'excommunication est prononcée contre tous ceux qui, sans excuse valable, manquent d'assister à la messe de paroisse trois dimanches consécutifs $\gg .{ }^{31}$ Après une très longue dissertation sur le vice de l'usure, Mgr de St-Vallier prévoit la peine d'excommunication contre ceux

${ }^{27}$ Ce Paul de Montéléon, fils du bouteiller du roi, était officier, prince du comté de Nice et parent de Pontchartrain. Il mourut avant 1717 et sa veuve se remaria à Ignace Aubert de La Chenaie. Quand il s'était présenté devant le Grand vicaire Grandelet, celui-ci avait exigé un certificat de liberté pour l'autoriser à se marier avec la fille de Lestringan de St-Martin, capitaine des gardes du château. Ils ne parvinrent pas à s'entendre, parce que de Montéléon ne pouvait produire ce papier officiel. Ils finirent par s'engueuler !... Or, le 7 janvier suivant, le Curé Boulard officiait à un mariage. Après la Consécration, de Montéléon et sa future l'interpellent à haute voix et lui demandent de les marier et disent qu'ils se prennent pour époux !... Le Curé Boulard, aussitôt après la messe dresse un procèsverbal de l'affaire et l'envoie à Raudot, l'intendant, lui demandant réparation. De Montéléon et sa femme finirent par se rendre; les bans furent publiés à Beauport, le 15 février, et le mariage eut lieu le lendemain. Gosselin, L'Eglise du Canada, IV; 342-347. - D'autres mariages de ce genre sont signalés le 28 fév. 1724, le 9 fév. 1751, le 15 fév. 1754. Et de Gaspé en signale un bon exemple, dans ses Mémoires, Cf. Roy, Les petites choses de notre histoire, $\mathrm{I}: 181-188$.

28 Gosselin, op. cit., 114s.

29 Ibid., 394s. Il s'agit de Jean-Baptiste d'Ailleboust des Musseaux (1666-1730), lieutenant réformé, cultivateur et commerçant.

30 Ibid., 395.

31 Le 22 déc. 1697, Mandements, I: 361. 
qui s'y livrent. ${ }^{32}$ On n'épargne pas cette sérieuse pénitence aux personnes du sexe qui sortiront et viendront à l'église avec des toilettes provocantes, ou même qui se baladeront, ainsi costumées, dans leur propre demeure. ${ }^{33}$ Défense aussi, sous la même peine, d'ondoyer à la maison les enfants nouveau-nés. ${ }^{34}$ Après le terrible incendie de Montréal, si nombreux et si honteux avaient été les pillages, que $\mathrm{Mgr}$ de St-Vallier se voit contraint d'excommunier tous les coupables qui ne veulent pas remettre les biens mal acquis, de même que les témoins qui ne dénonceraient pas les coupables. ${ }^{35}$

Tout ceci fait une longue liste de cas d'excommunication; s'il était bon de lui réserver cet espace dans le présent travail, c'est à cause de son importance comme témoignage sur les mœurs et la mentalité des gens au siècle que nous étudions. D'ailleurs, même les enfants, témoins plus ou moins conscients de ces violations graves des lois de l'Église, ne devaient-ils pas apprendre, et réciter tous les jours, ce $8 \mathrm{e}$ et $9 \mathrm{e}$ commandements de l'Église, pour éviter un danger qui les guetterait, devenus adultes !

\section{CHAPITRE III}

\section{MGR DE ST-VALLIER FUT-IL JANSÉNISTE OU SIMPLEMENT RIGORISTE?}

A lire les pages précédentes, il se peut qu'on garde difficilement un souvenir sympathique de Mgr de St-Vallier. Si dans les écrits qu'il nous a laissés, on ne regarde que ce côté des défenses et des peines ecclésiastiques qu'il sut fulminer contre les délinquants, on peut croire au fait d'un esprit à teinte janséniste, héritier de l'hérésie néfaste qui s'insinuait encore en France, à cette époque. ${ }^{36}$ Pourtant, il n'en est rien. Et sans

32 Mandements, I : 363-365.

33 Ibid., 365s.

34 Gosselin, Vie de Mgr de Laval, II: 43s, et Mandement du 5 fév. 1677, dans Mandements, I: 100 .

${ }^{35}$ Le 26 juin 1721, Mandements, $I: 501 \mathrm{~s}$.

$36 \mathrm{Au}$ temps où la querelle janséniste a l'envergure d'une crise nationale, Mgr Languet, d'abord évêque de Soissons, tient la tête de l'opposition. Il publie son Catéchisme diocésain, qu'il apporte avec lui, à Sens (d'où: 
faire sur ce propos une longue dissertation ou mise au point, il convient de relever la mémoire de ce saint Prélat; après quoi, on lui conservera tout au plus le seul qualificatif qu'il mérite à cet égard: RIGORISTE.

Ce qui, surtout, justifie cette position, ce sont ses œuvres elles-mêmes, d'autant qu'il serait de mauvaise psychologie de confondre caractère et enseignement. Une autorité peut être sévère; elle peut s'imposer sous des dehors rébarbatifs, et même éclater en maximes rigoureuses contre des abus, ou ce qu'elle croit tel, et, du même coup, professer une doctrine absolument orthodoxe. La personnalité d'un individu importe, sans doute, mais elle ne tient pas uniquement à la doctrine qu'il professe; c'est dire qu'on peut être violent, querelleur, intransigeant, qu'on peut même recourir aux grands moyens, sans pour cela tomber dans l'erreur ou l'hérésie doctrinale. Or, dans le cas de Mgr de St-Vallier, je crois qu'il faut intelligemment faire cette distinction, ou ces distinctions; autrement, le souvenir que l'esprit peut garder du personnage historique sera faussé par des apparences qui voilent la beauté de son zèle et la saine doctrine qu'il croyait et voulait enseigner à ses ouailles. Et je répète qu'il suffit de lire son œuvre écrite pour s'en rendre compte et pour corriger l'impression choquante que nous laisserait telle ou telle épisode disgracieuse de sa vie. Etre justifiable de lui attribuer des comportements outrés et de critiquer certaines de ses mesures administratives, n'amène en rien le droit de contester son attachement à l'Église de Rome et sa fidélité absolue à l'enseignement de celle-ci. ${ }^{37}$

le Catéchisme de Sens). Mgr Caylus, pro-janséniste, son subalterne, lui livre combat. Mgr Languet et son Catéchisme sortent avec les honneurs de la saine doctrine. Les Prélats dévoués à la cause de l'orthodoxie s'empressent de l'adopter. Hézard, Histoire du Catéchisme, etc., 212. Voir aussi, La Grande Encyclopédie, art. Beaumont (Christophe).

37 Les doutes sur l'orthodoxie de la doctrine de Mgr de St-Vallier viennent, du plus loin qu'on peut les retracer, de la critique de ses deux œuvres maîtresses, par le Père Bouvart, s.j., dès leur arrivée au pays. Ce P. Bouvart était alors supérieur des missions des Jésuites au Canada (1698-1704). Par souci de rendre service à Mgr de St-Vallier, son grand ami, il releva, en une cinquantaine de pages, tout ce qui, à son sens, méritait une critique dans les deux volumes doctrinaux. Mgr de St-Vallier, très blessé de ces observations franches, soumet le plaidoyer du P. Bouvart à la Sorbonne, pour vérification. Celle-ci donne une réponse cassante contre 
Dans son Rituel, prenons la partie qui traite de la Pénitence. Elle est cousue de citations des Conciles et des Saints-Pères. Il est facile de constater combien il tient à appuyer chaque prescription, chaque défense, comme aussi chaque exhortation, sur l'autorité de ces suprêmes magistères. Dans la seule partie que nous étudions, Mgr de St-Vallier cite environ 20 fois les Conciles ${ }^{38}$, et une dizaine de fois les Pères de l'Église. ${ }^{39}$ En si bonne compagnie, il était difficile de fausser l'enseignement traditionnel de l'Église romaine et d'introduire au pays une doctrine entachée de jansénisme et donc, en contradiction avec celle de Rome. Argumentation très faible, sans doute, mais continuons. En parlant du Grand Séminaire, il écrit que «le clergé n'est pas fort nombreux à Québec, mais on élève dans la cléricature, selon l'esprit du saint Concile de Trente, plusieurs enfants du pays $\gg .{ }^{40}$ Et quand, dans une Circulaire écrite avant son départ pour la France, Monseigneur donne ses recommandations, il insiste: "Comme le plus grand bien que nous puissions procurer à ce Diocèse en partant, est la bonne administration du Sacrement de Pénitence, nous croyons ne pouvoir mieux y réussir qu'en vous engageant chacun en particulier à lire attentivement la Session 14 du Concile de Trente, où toute la doctrine de ce Sacrement est contenu... ${ }^{41}$

Mgr Gosselin, son biographe, a soin de nous signaler souvent l'accusation implicite de jansénisme portée contre son héros; mais chaque fois, il répond péremptoirement à ces accusateurs inconnus. "Avant son élection, écrit-il, on rapportait que Mgr

Bouvart; elle l'accuse d'avoir insinué que Mgr de St-Vallier était suspect de pélagianisme, de luthéranisme, de calvinisme et de jansénisme; c'est une critique téméraire, scandaleuse et tendant au schisme, car le Rituel est très orthodoxe. On sait que Mgr de St-Vallier donna indirectement et partiellement raison au P. Bouvart, car il tient compte d'un bon nombre de ses remarques dans la seconde édition de son Rituel. Pour l'exposition résumée de cette critique de Bouvart, voir Gosselin, «Le Rituel de Mgr de StVallier », dans MSRC, (déc. 1914) : 251-257.

38 Il cite les Conciles, au moins aux pages suivantes: Rituel, 1ère éd., $84,85,88,90,91,92,93,100,105,107,109,112,115,117,120,125-130$.

$39 \mathrm{Il}$ cite les Pères, au moins aux pages suivantes: Ibid., 80, 83, 92, $93,96,100,107,111$.

40 Mandements, I: 193s ; Porter, L'Institution catéchistique, 20.

41 Mandements, I: 283. 
de St-Vallier était suspect de jansénisme ${ }^{42}$; pourtant, on pourrait dire de lui comme de Mgr de Laval: nul ne fut jamais moins janséniste que ce pieux évêque, qui, accoutumé lui-même, dès le bas âge, à la communion fréquente, savait mieux que personne et enseignait aux autres que c'est là le plus sûr moyen de se sanctifier. » 43 «Le Bréviaire, le Missel, le Cérémonial, le Rituel, tout était romain dans l'Église du Canada. ${ }^{44}$ M. Dudouyt, son mandaté auprès des autorités religieuses de Paris et du Séminaire des Missions-Etrangères, et même auprès du roi, avait horreur du jansénisme, et montrait tout le mal que l'hérésie faisait en France. ${ }^{45} \mathrm{Si}$, là-bas, Fleury fut l'ami de Bossuet et de Fénelon, Mgr de St-Vallier fut leur protégé. Sous ce patronage, ne voit-on pas qu'il aurait été de fort mauvais ton d'afficher des doctrines jansénistes ? ${ }^{46}$ L'Annaliste de l'Hôtel-Dieu de Québec apporte son témoignage en nous assurant «qu'il n'y a que cette misère, je veux dire l'erreur (janséniste), qui n'afflige pas le Canada ».47 Quand Mgr de St-Vallier se voit en droit de chasser le trop fameux Bénédictin défroqué, Georges-François Poulet, il publie, avec son Mandement de condamnation, la bulle Unigenitus de Clément XI ${ }^{48}$ contre le jansénisme, dont ce moine apportait, avec sa venue au pays, quelques mauvais germes. ${ }^{49}$

${ }^{42}$ Gosselin, Vie de Mgr de Laval, II: 343.

43 Ibid., 572 s.

44 C'est ce qui frappe Mgr de St-Vallier dès son arrivée à Québec. Ibid., 573 .

45 Ibid., 229.

46 Porter, L'Institution catéchistique, $165 \mathrm{~s}$.

47 Gosselin, Vie de Mgr de St-Vallier, I: 401.

$48 \mathrm{Cf}$. « Du «cas de conscience 》 à la Bulle Unigenitus», dans Jarry Preclin, Histoire de l'Eglise depuis les origines jusqu'à nos jours, XIX (1955) : 215-220.

49 Têtu, Notices biographiques, Les Evêques de Québec, 146. - Ce Père Poulet était devenu l'ami du fameux P. Quesnel, en exil à Amsterdam; c'est à ce contact qu'il devint peu à peu janséniste. Comme on voulait le renfermer dans un monastère, il se sauva au Canada, en 1714. Devenu malade, il est hospitalisé à l'Hôtel-Dieu; on essaie, sans succès, de le faire renoncer à ses erreurs. Il est chassé en Europe par les autorités religieuses et civiles. Il se réfugie en Hollande, en 1718, où il meurt après s'être plaint des traitements qu'il avait reçus au Canada. Roy, Les petites choses de notre histoire, V: 163-165. Gosselin, Vie de Mgr de St-Vallier, 325-331. Mgr Dosquet profite de sa visite au Canada pour faire signer à tous ses prêtres leur adhésion à la condamnation par Alexandre VII, du livre de Jansénius, l'Augustinus, et leur faire prêter serment à la Constitution Unigenitus de Clément XI, condamnant 101 propositions jansénistes du livre du Père Quesnel. Les chanoines l'avaient fait ensemble, le 3 nov. 1730. Gosselin, L'Eglise du Canada, IV: 139. 
Même surveillance exercée auprès du fameux abbé DominiqueMarie Varlet, janséniste d'esprit, qui avait réussi à s'insinuer au pays en 1712. Il devait bientôt retourner en Europe, où il mourut en 1742, après avoir été excommunié par trois papes. ${ }^{50}$

On peut apprécier l'esprit de docilité de Mgr de St-Vallier à l'esprit de Rome, au sujet, par exemple, de la réception des sacrements. Un janséniste authentique, lui, aurait prêché ce que l'on sait: l'éloignement de la sainte Table et du confessionnal; alors que Mgr de St-Vallier exhorte les fidèles à la pratique fréquente des sacrements; et à maintes reprises il recommande la communion au moins une fois tous les mois. ${ }^{51}$ Cette clause de la communion mensuelle paraît dans les règlements de la Société de la Ste-Famille ${ }^{52}$. Dans le récit d'un de ses voyages jusqu'aux confins de son immense diocèse, il assure que les sauvages eux-mêmes se confessent tous les mois, et la plupart le font de 15 jours en 15 jours. ${ }^{53}$ On ne comprend pas qu'un Jésuite de Montréal ait osé l'accuser «d'ôter les communions générales et fréquentes ... ${ }^{54}$; il suffit de lire les Mandements pour juger de cette accusation. S'il exhorte les confesseurs à se montrer sévères pour absoudre des péchés, il insiste pour que ce ne soit jamais au-delà des limites d'une saine théologie..55

Un son de cloche identique est donné par cet abbé Navières, curé à Ste-Anne-de-Beaupré, de 1734 à 1740. Il écrit à l'un de ses amis de Limoges: «Les confessions et communions sont si

50 Gosselin, Vie de Mgr de St-Vallier, I : 333-335.

51 Ibid., 33, 98; Mandements, I: 332. - Il est vrai que Bouvart s'était cru justifié de dire que «la seconde réflexion est que quoique la spéculation et en parlant en général il ( $\mathrm{Mgr}$ de St-Vallier) autorise la confession fréquente et la fréquente communion, il les ôte en effet par les difficultés qu'il y met dans la pratique .... Gosselin, «Le Rituel de Mgr de StVallier », dans MSRC (déc. 1914) : 225. Un peu dans le même ordre d'idées, Bouvart parle des billets de confession surtout pour le temps de Pâques, et affirme que Mgr de St-Vallier se contredit: il l'exige avec précision, dit-il, puis, ailleurs il (toujours Mgr) déclare nécessaire que les curés soient faciles à les donner, et ailleurs encore il ordonne au pénitent de rapporter son billet au curé, s'il a été à confesse à un autre. Gosselin, op. cit., 255. 52 Mandements, I: 60 .

53 Mandements, I: 230ss. - L'on trouve la relation complète du voyage de Mgr de St-Vallier à travers son diocèse, dans Mandements, I: 191-265.

54 Ibid., 209. Cf. Relations des Jésuites, éd. Burrows, 64: 118.

55 Gosselin, L'Eglise du Canada, IV: 73. 
fréquentes que je ne crois pas qu'il y ait en France de paroisse de campagne où elles soient plus communes. Les gens me tiennent au confessionnal, le dimanche pendant quatre heures, tellement que je dois en envoyer plusieurs pour célébrer ma messe, car les autres se plaignent d'attendre trop longtemps. Si nous étions trois ou quatre, nous aurions suffisamment d'occupations. ${ }^{56}$ Mgr de St-Vallier était mort à cette époque, mais peut-on croire qu'une telle ferveur se fût spontanément allumée dès sa disparition?

Cette argumentation, par le dehors je dirais, n'est pas serrée; les textes et les exemples cités ne règlent pas à eux seuls le problème, ni ne donnent la réplique finale à un accusateur entêté qui voudrait absolument que Mgr de St-Vallier ait été janséniste ! Mais cependant, il ne faut rien tirer par les cheveux, et, plutôt que de tabler sur une opinion sans fondements, qui ne répète que les échos venant d'un passé mal connu, mieux vaut aller froidement aux sources qui ont conservé la doctrine même de Mgr de St-Vallier; il y a déversé son esprit, dans un enseignement lumineux et en tout point inattaquable, quant à son orthodoxie. L'étude plus poussée de ce problème théologico-historique ne manquerait pas d'intérêt; mais alors, il faudrait pour la mener à des conclusions encore plus sûres, poser des bases doctrinales solides et bien définir les termes, comme jansénisme, rigorisme, et autres; ce serait un premier terrain d'entente que de savoir de quoi il s'agit et d'appeler les choses par leur nom, afin de discuter sur le même plan. Laissons à un autre de reprendre cette étude où, sans doute, il nous brosserait du même coup un tableau d'ensemble de ce que pouvait être la mentalité religieuse de nos ancêtres et celle du clergé qui, sous le régime français, la leur avait inspirée par le prône, le catéchisme, les conseils et les exemples vécus.

Quant à Mgr de St-Vallier, on peut dire, sans exagérer, qu'aucun Évêque ne poussa plus loin que lui l'amour de la discipline ecclésiastique; on peut aussi le regarder comme le législateur de celle qui régit encore aujourd'hui le clergé canadien,

56 Ibid., V : 338s. 
compte tenu des mises au point faites par le Code de Droit canonique de 1917. Pour comprendre Mgr de St-Vallier dans sa personne et dans son œuvre, il faut lire son Rituel, les Ordonnances des quatre Synodes qu'il présida, son Catéchisme, et surtout ses nombreux Mandements. ${ }^{57}$

On concèdera, alors, qu'il ait été RIGORISTE, un peu à la façon du grand et célèbre réformateur saint Charles Borromée, son modèle; mais on se refusera à croire qu'il ait été le moindrement JANSÉNISTE, dans le vrai sens du mot.

\section{CONCLUSION}

Sous le régime français, nous avons eu un enseignement doctrinal sur la Confession; cet enseignement s'est avéré ferme dès les premières directives venant de Mgr de Laval. Sous son épiscopat pourtant, l'uniformité de l'enseignement, qu'il vînt du clergé ou des parents, fit défaut, parce que les sources d'informations manquaient elles-mêmes d'unité. Dans son enthousiasme et son zèle éclairé, Mgr de St-Vallier jugea urgent de fournir à ses ouailles un corps de doctrine d'inspiration franchement catholique-romaine. A cet effet, il publia, coup sur coup, son Rituel et son Catéchisme. Pour compléter ses propres écrits et en donner, au besoin, une interprétation authentique, il lança à la surface de son grand diocèse, Mandements et Ordonnances, et réunit des Synodes à quatre reprises. En tous ces écrits doctrinaux et disciplinaires, il venait avec fermeté, sur un ton souvent tranchant, lever des doutes et fixer à ses confesseurs et catéchistes, une ligne de conduite, dans leur tâche, parfois ardue, de guider les consciences.

L'introduction, vers 1750, du Catéchisme de Mgr Languet n'amena aucune révolution dans l'enseignement de la Pénitence, puisque sa doctrine reproduisait pour ainsi dire, l'œuvre magistrale du grand pionnier de la catéchistique chez nous. Mgr Briand et ses successeurs, à diverses reprises, réimprimèrent la même doctrine, celle même qui s'est transmise jusqu'à nos jours, par toutes les voies de l'enseignement religieux.

57 Têtu, Notices biographiques. Les Evêques de Québec, 147. 
Le fond de cette doctrine, non seulement formait un tout bien organisé, mais il reflétait - du moins en ce qui regarde la Pénitence - une soumission totale à l'Église de Rome, et un peu aussi, la mentalité de ceux qui avaient mission de catéchiser le peuple. Or, s'il est assez évident que certaines règles disciplinaires, concernant la confession, fussent d'inspiration rigoriste, il faut en attribuer la cause, surtout à l'état général des esprits, aux $17 \mathrm{e}$ et $18 \mathrm{e}$ siècles. De plus, nos Évêques furent des clercs, disciples de l'École française d'ascétisme, généralement formés dans le camp opposé à celui des Jansénistes. Ils gardaient les principes d'une forte ascèse et les dehors d'une austérité de bon aloi, qu'une étude, même superficielle de cette période, en Europe, nous fait mieux comprendre. Nos pères, héritiers de cet esprit, disons rigoriste, connurent les défauts de leur temps, mais vivaient quand même un christianisme fort simple et assez évangélique.

Le rappel fréquent aux refus d'absolution et aux excommunications souligne seulement le dicton: autres temps, autres mœurs ! Pruderie, retenue, quelquefois scrupule: allures timorées de tout un peuple, qui dénotent de quelle verte façon il avait été éduqué, dès l'origine! Même le peu qui nous reste encore de ces habitudes austères fait honneur aux devanciers qui s'étaient mis docilement à l'école de leurs pasteurs. En ceci, pourtant, il faut retenir son pathos et ne rien exagérer. De récents historiens ne refusent-ils pas volontiers à nos ancêtres la gloire - car c'en est une - de nous avoir donné, par leur conduite, en général, de bonnes leçons de savoir-vivre chrétien ? Une forte discipline de la Confession explique, en partie, cette formation d'une conscience collective opposée à ce qu'on appellerait le laxisme religieux, ${ }^{58}$

Si quelques-unes des considérations précédentes ne se dégagent pas directement de notre étude, elles y sont sous-jacentes, et on les sent qui s'imposent, à toutes les phases de l'exposition. Ces problèmes, autant du domaine de la théologie que de celui de l'histoire, demanderaient, pour être résolus de façon satis-

${ }^{58}$ Vacant-Mangenot, Dictionnaire de Théologie catholique, IX, au mot laxisme, Col. 37-86. 
faisante aux yeux de l'historien et du théologien, que les composantes de la mentalité religieuse de notre peuple soient repensées, au cours d'une étude plus vaste qui embrasserait, sous tous les angles, les faits attestés par les documents: large synthèse, dans laquelle le sacrement de Pénitence, comme ceux de l'Eucharistie et du Mariage, apparaîtrait, sans doute, comme un facteur de première importance. Souhaitons que se poursuivent ces recherches, et qu'elles soient menées à bonne fin, par un esprit judicieux, au bénéfice de la vérité historique.

VALÉRIEN ROY

Sherbrooke, Québec.

P.S. - Faute d'espace, nous avons dû reporter à notre numéro de juin, l'article de M. Robert Le Blant, « La famille Boullé, 1586-1639 ». Il en est de même des comptes rendus de volumes arrivés trop tard. 